\title{
DEGENERATIONS OF GRADED COHEN-MACAULAY MODULES
}

\author{
NAOYA HIRAMATSU
}

\begin{abstract}
We introduce a notion of degenerations of graded modules. In relation to it, we also introduce several partial orders as graded analogies of the hom order, the degeneration order and the extension order. We prove that these orders are identical on the graded Cohen-Macaulay modules over a graded ring which is of graded finite representation type and representation directed.
\end{abstract}

1. Introduction. The notion of degenerations of modules appears in geometric methods of representation theory of finite-dimensional algebras. In [17], Yoshino gives a scheme-theoretical definition of degenerations, so that it can be considered for modules over a Noetherian algebra which is not necessarily finite dimensional. Now, a theory of degenerations is considered for derived module categories [11] or stable module categories [18]. The degeneration problem of modules has been studied by many authors $[\mathbf{6}, \mathbf{1 4}, \mathbf{1 6}, \mathbf{1 7}, \mathbf{2 0}, \mathbf{2 1}]$. For the study, several order relations for modules, such as the hom order, the degeneration order and the extension order, were introduced, and the connection among them has been studied. In the previous paper [9], the author gives the complete description of degenerations over a ring of even-dimensional simple hypersurface singularity of type $\left(A_{n}\right)$.

In the present paper, we consider degenerations of graded CohenMacaulay modules over a graded Gorenstein ring with a graded isolated singularity. First we consider an order relation on a category of graded Cohen-Macaulay modules which is called the hom order (Definition 2.2 ). We shall show that it is actually a partial order if a graded ring is Gorenstein with a graded isolated singularity.

2010 AMS Mathematics subject classification. Primary 16W50, Secondary $13 \mathrm{D} 10$.

Keywords and phrases. degeneration, graded Cohen-Macaulay module, finite representation type.

Received by the editors on May 14, 2013, and in revised form on February 11, 2014.

DOI: $10.1216 / J C A-2015-7-2-221$

Copyright (C)2015 Rocky Mountain Mathematics Consortium 
In Section 3, we propose a definition of degenerations for graded modules (Definition 3.1) and we state several properties of it. We show that, if the graded ring is of graded finite representation type and representation directed, then the hom order, the degeneration order and the extension order are identical on the graded Cohen-Macaulay modules. We also consider a stable analogue of degenerations of graded Cohen-Macaulay modules in Section 4.

2. Hom order on graded modules. Throughout the paper, let $R=\oplus_{i=0}^{\infty} R_{i}$ be a commutative Noetherian $\mathbb{N}$-graded Cohen-Macaulay ring with $R_{0}=k$ a field of characteristic zero. A graded ring is said to be *local if the set of graded proper ideals has a unique maximal element. Thus, $R$ is ${ }^{*}$ local since $\mathfrak{m}=\oplus_{i>0} R_{i}$ is a unique maximal ideal of $R$. We denote by $\bmod ^{\mathbb{Z}}(R)$ the category of finitely generated $\mathbb{Z}$-graded modules whose morphisms are homogenous morphisms that preserve degrees. For $i \in \mathbb{Z}, M(i) \in \bmod ^{\mathbb{Z}}(R)$ is defined by $M(i)_{n}=$ $M_{n+i}$. Then $\operatorname{Hom}_{R}(M, N(i))$ consisting of homogenous morphisms of degree $i$, and we set

$$
{ }^{*} \operatorname{Hom}_{R}(M, N)=\oplus_{i \in \mathbb{Z}} \operatorname{Hom}_{R}(M, N(i)) .
$$

For a graded prime ideal $\mathfrak{p}$ of $R$, we denote by $R_{(\mathfrak{p})}$ a homogenous localization of $R$ by $\mathfrak{p}$ (see [7, subsection 1.5.]). For $M \in \bmod ^{\mathbb{Z}}(R)$, take a graded free resolution

$$
\cdots \rightarrow F_{l} \stackrel{d_{l}}{\rightarrow} F_{l-1} \rightarrow \cdots \rightarrow F_{1} \stackrel{d_{1}}{\rightarrow} F_{0} \rightarrow M \rightarrow 0 .
$$

We define an $l$ th syzygy module $\Omega^{l} M$ of $M$ by $\operatorname{Im}\left(d_{l}\right)$. We say that a graded $R$-module $M$ is said to be a graded Cohen-Macaulay $R$-module if

$$
{ }^{*} \operatorname{Ext}_{R}^{i}(R / \mathfrak{m}, M)=0 \quad \text { for any } i<d=\operatorname{dim} R .
$$

In particular, this condition is equivalent to

$$
{ }^{*} \operatorname{Ext}_{R}^{i}\left(M, \omega_{R}\right)=0 \text { for any } i>0,
$$

where $\omega_{R}$ is a ${ }^{*}$ canonical module of $R$. The notion of a ${ }^{*}$ canocial module is a graded version of a canonical module. In our setting, *canocial module is a canonical module of $R$, and we can take ${ }^{*} \operatorname{Hom}_{R}\left({ }^{*} \mathrm{H}_{\mathfrak{m}}^{d}(R), k\right)$ as $\omega_{R}$ where ${ }^{*} \mathrm{H}_{\mathfrak{m}}^{d}(R)=\underline{\lim ^{*}} \operatorname{Ext}_{R}^{d}\left(R / \mathfrak{m}^{k}, R\right)$. See [7, subsection 3.6.] for details. We denote by $\mathrm{CM}^{\mathbb{Z}}(R)$ the full subcategory of 
$\bmod ^{\mathbb{Z}}(R)$ consisting of graded Cohen-Macaulay $R$-modules. By our assumption on $R, \bmod ^{\mathbb{Z}}(R)$ and $\mathrm{CM}^{\mathbb{Z}}(R)$ are Krull-Schmidt, namely, each object can be decomposed into indecomposable objects up to isomorphism uniquely. For $M \in \bmod ^{\mathbb{Z}}(R)$, we denote by $h(M)$ a sequence $\left(\operatorname{dim}_{k} M_{n}\right)_{n \in \mathbb{Z}}$ of non-negative integers. By the definition, it is easy to see that $h(M)=h(N)$ if and only if they have the same Hilbert series. Moreover, we also have that $h(M)=h(N)$ if and only if $h\left(M^{*}\right)=h\left(N^{*}\right)$ where $(-)^{*}={ }^{*} \operatorname{Hom}_{R}\left(-, \omega_{R}\right)$. See [7, Theorem 4.4.5.].

Remark 2.1. For a short exact sequence of finitely generated graded $R$-modules $0 \rightarrow X \rightarrow Y \rightarrow Z \rightarrow 0$, we see that $\operatorname{dim}_{k} Y_{n}=\operatorname{dim}_{k} X_{n}+$ $\operatorname{dim}_{k} Z_{n}$ for each $n \in \mathbb{Z}$. If $M$ and $N$ give the same class in the Grothendieck group, i.e., $[M]=[N]$ as an element of $K_{0}\left(\bmod ^{\mathbb{Z}}(R)\right)$, then there exists a short exact sequence $0 \rightarrow X \rightarrow Y \rightarrow Z \rightarrow 0$ such that $M \oplus X \oplus Z \cong N \oplus Y$ by the definition. Therefore, we have $h(M)=h(N)$ if $[M]=[N]$ in $K_{0}\left(\bmod ^{\mathbb{Z}}(R)\right)$. However, the converse does not hold in general. Let $R=k[x, y] /\left(x^{2}-y^{2}\right)$ with $\operatorname{deg} x=\operatorname{deg} y=1$, and set $M=R /(x+y)$ and $N=R /(x-y)$. Then $h(M)=h(N)$ and $[M] \neq[N]$ in $K_{0}\left(\bmod ^{\mathbb{Z}}(R)\right)$. In fact, let $\mathfrak{p}$ be an ideal $(x+y) R$. Then $\operatorname{rank}_{R_{(\mathfrak{p})}} M_{(\mathfrak{p})}=1$ and $\operatorname{rank}_{R_{(\mathfrak{p})}} N_{(\mathfrak{p})}=0$. Note that $[M]=[N]$ on $K_{0}\left(\bmod ^{\mathbb{Z}}\left(R_{(\mathfrak{p})}\right)\right)$ yields that $\left[M_{(\mathfrak{p})}\right]=\left[N_{(\mathfrak{p})}\right]$ on $K_{0}\left(\bmod ^{\mathbb{Z}}\left(R_{(\mathfrak{p})}\right)\right)$. Thus, $[M]=[N]$ can never happen.

Our motivation of the paper is to investigate the graded degenerations of graded Cohen-Macaulay modules in terms of some order relations. For this reason, we consider the following relation on $\mathrm{CM}^{\mathbb{Z}}(R)$ that is known as the hom order.

Definition 2.2. For $M, N \in \mathrm{CM}^{\mathbb{Z}}(R)$, we define $M \leq_{\text {hom }} N$ if $[M, X] \leq$ $[N, X]$ for each $X \in \mathrm{CM}^{\mathbb{Z}}(R)$. Here $[M, X]$ is an abbreviation of $\operatorname{dim}_{k} \operatorname{Hom}_{R}(M, X)$.

Remark 2.3. For $M, N \in \bmod ^{\mathbb{Z}}(R),[M, N]$ is finite, and thus we can consider the above relation. Let $k$ be a commutative ring and $\mathcal{A}$ an abelian $k$-category such that each morphism set has finite length as a $k$ module. As shown in [5], $\leq_{\text {hom }}$ is a partial order on a full subcategory of $\mathcal{A}$ which is closed under direct sums and kernels. Thus, if $R$ is of 
dimension 0,1 or $2, \leq_{\text {hom }}$ is a partial order on $\mathrm{CM}^{\mathbb{Z}}(R)$ since $\mathrm{CM}^{\mathbb{Z}}(R)$ is closed under direct sums and kernels in such cases.

Lemma 2.4. Let $R$ be a graded Gorenstein ring, and let $M$ and $N$ be graded Cohen-Macaulay $R$-modules. Suppose that $h(M)=h(N)$. For $Y \in \bmod ^{\mathbb{Z}}(R)$ which is of finite projective dimension, we have $[M, Y]=[N, Y]$.

Proof. For a graded $R$-module $Y$ which is of finite projective dimension, $\Omega^{i} Y$ is also of finite projective dimension. Since $R$ is Gorenstein, we have ${ }^{*} \operatorname{Ext}_{R}^{1}\left(M, \Omega^{i} Y\right)=0$ for all graded Cohen-Macaulay $R$ modules $M$. Thus, taking a graded free resolution of $Y$ and applying $\operatorname{Hom}_{R}(M,-)$ to the resolution, we get an exact sequence

$$
\begin{aligned}
0 \longrightarrow \operatorname{Hom}_{R}\left(M, F_{l}\right) & \longrightarrow \operatorname{Hom}_{R}\left(M, F_{l-1}\right) \longrightarrow \cdots \\
& \longrightarrow \operatorname{Hom}_{R}\left(M, F_{0}\right) \longrightarrow \operatorname{Hom}_{R}(M, Y) \longrightarrow 0 .
\end{aligned}
$$

Hence,

$$
[M, Y]=\sum_{i=0}^{l}(-1)^{i}\left[M, F_{i}\right]
$$

We also have

$$
[N, Y]=\sum_{i=0}^{l}(-1)^{i}\left[N, F_{i}\right]
$$

Since $R$ is Gorenstein, $\omega_{R}=R(l)$ for some $l \in \mathbb{Z}$. Then we have an equality $[M, F]=[N, F]$ for each graded free module. Therefore, we have

$$
[M, Y]=\sum_{i=0}^{l}(-1)^{i}\left[M, F_{i}\right]=\sum_{i=0}^{l}(-1)^{i}\left[N, F_{i}\right]=[N, Y]
$$

In this paper, we use the theory of Auslander-Reiten (AR) sequences of graded Cohen-Macaulay modules. For details, we recommend $[\mathbf{3}, \mathbf{4}$, 10] and [15, Chapter 15]. We denote by $\mathrm{CM}_{0}^{\mathbb{Z}}(R)$ the full subcategory of $\mathrm{CM}^{\mathbb{Z}}(R)$ consisting of $M \in \mathrm{CM}^{\mathbb{Z}}(R)$ such that $M_{(\mathfrak{p})}$ is $R_{(\mathfrak{p})}$-free for any graded prime ideal $\mathfrak{p} \neq \mathfrak{m}$.

Theorem 2.5. $[\mathbf{3}, \mathbf{4}, \mathbf{1 5}, \mathbf{1 0}]$. Let $(R, \mathfrak{m})$ be a Noetherian $\mathbb{Z}$-graded Gorenstein *local ring. Then $\mathrm{CM}_{0}^{\mathbb{Z}}(R)$ admits $A R$ sequences. 
Definition 2.6. We say that $(R, \mathfrak{m})$ is a graded isolated singularity if each graded localization $R_{(\mathfrak{p})}$ is regular for each graded prime ideal $\mathfrak{p}$ with $\mathfrak{p} \neq \mathfrak{m}$.

It is easy to see that $\mathrm{CM}^{\mathbb{Z}}(R)=\mathrm{CM}_{0}^{\mathbb{Z}}(R)$ if $R$ is a graded isolated singularity, so that $\mathrm{CM}^{\mathbb{Z}}(R)$ admits $\mathrm{AR}$ sequences. We denote by $\mu(M, Z)$ the multiplicity of $Z$ as a direct summand of $M$.

Theorem 2.7. Let $R$ be a graded Gorenstein ring with $R_{0}=k$ an algebraically closed field, and let $M$ and $N$ be graded Cohen-Macaulay $R$-modules. Assume that $R$ is a graded isolated singularity. Then $[M, X]=[N, X]$ for each $X \in \mathrm{CM}^{\mathbb{Z}}(R)$ if and only if $M \cong N$. In particular, $\leq_{\text {hom }}$ is a partial order on $\mathrm{CM}^{\mathbb{Z}}(R)$.

Proof. We decompose $M$ as $M=\oplus M_{i}^{\mu\left(M, M_{i}\right)}$ where $M_{i}$ are indecomposable graded Cohen-Macaulay $R$-modules. If $M_{i}$ is not free, we can take the AR sequence ending in $M_{i}$

$$
0 \longrightarrow \tau M_{i} \longrightarrow E_{i} \longrightarrow M_{i} \longrightarrow 0
$$

where $\tau M_{i}$ is an AR translation of $M_{i}$. Apply $\operatorname{Hom}_{R}(M,-)$ and $\operatorname{Hom}_{R}(N,-)$ to the sequence. Since $k$ is an algebraically closed field, $\operatorname{End}_{R}(X) / \operatorname{rad}_{\operatorname{End}_{R}}(X) \cong k$ for each indecomposable graded CohenMacaulay module $X$. Hence, we have

$$
\begin{aligned}
0 \longrightarrow \operatorname{Hom}_{R}\left(M, \tau M_{i}\right) \longrightarrow & \operatorname{Hom}_{R}\left(M, E_{i}\right) \\
& \longrightarrow \operatorname{Hom}_{R}\left(M, M_{i}\right) \longrightarrow k^{\mu\left(M, M_{i}\right)} \longrightarrow 0
\end{aligned}
$$

and

$$
\begin{aligned}
0 \longrightarrow \operatorname{Hom}_{R}\left(N, \tau M_{i}\right) \longrightarrow & \operatorname{Hom}_{R}\left(N, E_{i}\right) \\
& \longrightarrow \operatorname{Hom}_{R}\left(N, M_{i}\right) \longrightarrow k^{\mu\left(N, M_{i}\right)} \longrightarrow 0
\end{aligned}
$$

(see [13, Corollary 13.17] or [15, Lemma 5.5]). Counting the dimensions of terms, we conclude that $\mu\left(M, M_{i}\right)=\mu\left(N, M_{i}\right)$.

If $M_{i}$ is free, we may assume that $M_{i}=R$. Let $\mathfrak{m}$ be a *maximal ideal of $R$. We consider the graded Cohen-Macaulay approximation (Remark 2.8) of $\mathfrak{m}$

$$
0 \longrightarrow Y \longrightarrow X \longrightarrow \mathfrak{m} \longrightarrow 0 \text {. }
$$


We note that $X$ is a graded Cohen-Macaulay $R$-module and $Y$ is of finite projective dimension. Let $f$ be a composition map of the approximation $X \rightarrow \mathfrak{m}$ and a natural inclusion $\mathfrak{m} \rightarrow R$. Then we get the following commutative diagram.

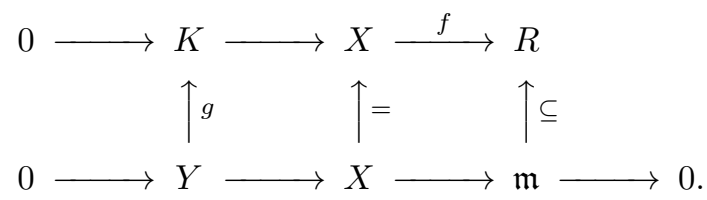

By a diagram chasing, we see that $g$ is surjective so that $K$ is of finite projective dimension. Now we obtain an exact sequence

$$
\begin{aligned}
0 \longrightarrow \operatorname{Hom}_{R}(M, K) & \longrightarrow \operatorname{Hom}_{R}(M, X) \\
& \longrightarrow \operatorname{Hom}_{R}(M, R) \longrightarrow k^{\mu(M, R)} \longrightarrow 0 .
\end{aligned}
$$

According to Lemma 2.4, $[M, K]=[N, K]$. Thus,

$$
[M, R]+[M, K]-[M, X]=[N, R]+[N, K]-[N, X] .
$$

Hence, $\mu(M, R)=\mu(N, R)$. Consequently $M \cong N$.

Remark 2.8. As mentioned in [2, Section 1] or [8, Theorem 1.12.10], the decomposition (approximation) theorem for an abelian category is given. If $R$ is Gorenstein, one can show that $\left(\mathrm{CM}^{\mathbb{Z}}(R), \operatorname{FPD}(R), \mathrm{F}(R)\right)$ is an Auslander-Buchweitz context in $\bmod ^{\mathbb{Z}}(R)$, where $\operatorname{FPD}(R)$ (respectively, $\mathrm{F}(R)$ ) is a full subcategory of $\bmod ^{\mathbb{Z}}(R)$ consisting of graded modules of finite projective dimension (respectively, graded free modules). See also [13, Theorem 11.17.]

\section{Graded degenerations of graded Cohen-Macaulay mod-} ules. We define a notion of degenerations for graded modules.

Definition 3.1. Let $R$ be a Noetherian $\mathbb{N}$-graded ring where $R_{0}=k$ is a field, and let $V=k[[t]]$ with a trivial gradation and $K$ be the localization by $t$, namely, $K=V_{t}=k(t)$. For finitely generated graded $R$-modules $M$ and $N$, we say that $M$ gradually degenerates to $N$ or $N$ is a graded degeneration of $M$ if there is a finitely generated graded $R \otimes_{k} V$-module $Q$ which satisfies the following conditions:

(1) $Q$ is flat as a $V$-module. 
(2) $Q \otimes_{V} V / t V \cong N$ as a graded $R$-module.

(3) $Q \otimes_{V} K \cong M \otimes_{k} K$ as a graded $R \otimes_{k} K$-module.

In [17], Yoshino gives a necessary and sufficient condition for degenerations of (non-graded) modules. One can also show its graded version in a similar way. See also $[\mathbf{1 4}, \mathbf{2 1}]$.

Theorem 3.2. [17, Theorem 2.2] The following conditions are equivalent for finitely generated graded $R$-modules $M$ and $N$.

(1) $M$ gradually degenerates to $N$.

(2) There is a short exact sequence of finitely generated graded $R$ modules

$$
0 \longrightarrow Z \longrightarrow M \oplus Z \longrightarrow N \longrightarrow 0
$$

Proof. We give the outline of the proof for the convenience of the reader.

$(1) \Rightarrow(2)$. Since $M \otimes_{k} V$ is a finitely generated graded $R \otimes_{k} V$ submodule of $M \otimes_{k} K \cong Q \otimes_{V} K$, we see that $M \otimes_{k} V \subseteq\left(1 / t^{n}\right) Q$ for large $n$. Note that $Q$ is $V$-flat and $\operatorname{deg} t=0$. We can replace $Q$ with $\left(1 / t^{n}\right) Q$, and we may assume that $M \otimes_{k} V \subseteq Q$ as a graded $R \otimes_{k} V$ module. Then we can show that $Q /\left(M \otimes_{k} t V\right) \cong M \oplus Q /\left(M \otimes_{k} V\right)$ and $Q /\left(M \otimes_{k} V\right)$ is finitely generated as a graded $R$-module. Take a graded $R$-homomorphism

$f: Q /\left(M \otimes_{k} V\right) \longrightarrow Q /\left(M \otimes_{k} t V\right): f\left(x+M \otimes_{k} V\right)=t x+M \otimes_{k} t V$.

One can show that $f$ is a monomorphism and Coker $(f) \cong N$, so that $f$ induces a sequence of graded $R$-modules

$$
0 \longrightarrow Z \longrightarrow M \oplus Z \longrightarrow N \longrightarrow 0,
$$

where $Z=Q /\left(M \otimes_{k} V\right)$.

$(2) \Rightarrow(1)$. Suppose that we have a short exact sequence

$$
0 \longrightarrow Z \stackrel{\left(\begin{array}{l}
\varphi \\
\psi
\end{array}\right)}{\longrightarrow} M \oplus Z \longrightarrow N \longrightarrow 0 .
$$

As remarked below (Remark $3.3(1)$ ), we may assume that the endomorphism $\psi$ of $Z$ is nilpotent. Note here again that $\operatorname{deg} t=0$. We 
consider the graded $R \otimes_{k} V$-homomorphism

$$
g=\left(\begin{array}{c}
\varphi \otimes(1-t) \\
1 \otimes t+\psi \otimes(1-t)
\end{array}\right): Z \otimes_{k} V \longrightarrow(M \oplus Z) \otimes_{k} V .
$$

We denote the cokernel of $g$ by $Q$. Then we can show that $Q$ is $V$-flat, $Q \otimes_{V} V / t V \cong N$ as a graded $R$-module and $Q \otimes_{V} K \cong M \otimes_{k} K$ as a graded $R \otimes_{k} K$-module. Namely, $M$ gradually degenerates to $N$.

\section{Remark 3.3.}

(1) As Yoshino has shown in [17], the endomorphism of $Z$ in the sequence of Theorem 3.2 is nilpotent. Note that we do not need the nilpotency assumption here. Actually, since $\operatorname{End}_{R}(Z)$ is Artinian, by using the Fitting theorem, we can describe the endomorphism as a direct sum of an isomorphism and a nilpotent morphism. See also [17, Remark 2.3.].

(2) Assume that $M$ and $N$ are graded Cohen-Macaulay modules. Then we can show that $Z$ is also a graded Cohen-Macaulay $R$-module. See [17, Remark 4.3.].

(3) Assume that there is an exact sequence of finitely generated graded $R$-modules

$$
0 \longrightarrow \mathrm{L} \longrightarrow \mathrm{M} \longrightarrow \mathrm{N} \longrightarrow 0 \text {. }
$$

Then $M$ gradually degenerates to $L \oplus N$. See [17, Remark 2.5], for instance.

(4) Let $M$ and $N$ be finitely generated graded $R$-modules, and suppose that $M$ gradually degenerates to $N$. Then the modules $M$ and $N$ give the same class in the Grothendieck group.

We can also prove in a similar way to the proof of [19, Theorem 2.1] that, for $L, M, N \in \bmod ^{\mathbb{Z}}(R)$, if $L$ gradually degenerates to $M$ and if $M$ gradually degenerates to $N$, then $L$ gradually degenerates to $N$. And one can show that, if $L$ gradually degenerates to $M$ then $L \leq_{\text {hom }} M$. Using this fact, we define partial orders as follows.

Definition 3.4. For finitely generated graded $R$-modules $M, N$, we define the relation $M \leq_{\operatorname{deg}} N$, which is called the degeneration order, if $M$ gradually degenerates to $N$. We also define the relation $M \leq_{\operatorname{ext}} N$ if there are modules $M_{i}, N^{\prime}{ }_{i}, N^{\prime \prime}{ }_{i}$ and short exact sequences $0 \rightarrow N^{\prime}{ }_{i} \rightarrow$ 
$M_{i} \rightarrow N^{\prime \prime}{ }_{i} \rightarrow 0$ in $\bmod ^{\mathbb{Z}}(R)$ so that $M=M_{1}, M_{i+1}=N^{\prime}{ }_{i} \oplus N^{\prime \prime}{ }_{i}$, $1 \leq i \leq s$ and $N=M_{s+1}$ for some $s$.

For $M \in \mathrm{CM}^{\mathbb{Z}}(R)$, we take a first syzygy module of $M^{*}$

$$
0 \longrightarrow \Omega^{1} M^{*} \longrightarrow F \longrightarrow M^{*} \longrightarrow 0 \text {. }
$$

Applying ${ }^{*} \operatorname{Hom}_{R}\left(-, \omega_{R}\right)$ to the sequence, we have

$$
0 \longrightarrow M^{* *} \cong M \longrightarrow F^{*} \longrightarrow\left(\Omega^{1} M^{*}\right)^{*} \longrightarrow 0 .
$$

Then we denote $\left(\Omega^{1} M^{*}\right)^{*}$ by $\Omega^{-1} M$.

Lemma 3.5. Let $R$ be a graded isolated singularity with a ${ }^{*}$ canonical module $\omega_{R}$, and let $M$ and $N$ be graded Cohen-Macaulay $R$-modules. Assume that $h(M)=h(N)$ and $M \leq_{\text {hom }} N$. Then, for each graded Cohen-Macaulay $R$-module $X$, there exists an integer $l_{X} \gg 0$ such that $\left[M, X\left( \pm l_{X}\right)\right]=\left[N, X\left( \pm l_{X}\right)\right]$.

Proof. For each $X \in \mathrm{CM}^{\mathbb{Z}}(R)$, we can take an exact sequence as above

$$
0 \longrightarrow X \longrightarrow E \longrightarrow \Omega^{-1} X \longrightarrow 0 \text {. }
$$

Note that $E$ is a direct sum of $\omega_{R}(n)$ for some integers $n$. Applying ${ }^{*} \operatorname{Hom}_{R}(M,-)$ to the sequence, we have

$$
\begin{aligned}
0 \longrightarrow{ }^{*} \operatorname{Hom}_{R}(M, X) & \longrightarrow{ }^{*} \operatorname{Hom}_{R}(M, E) \\
& \longrightarrow{ }^{*} \operatorname{Hom}_{R}\left(M, \Omega^{-1} X\right) \longrightarrow{ }^{*} \operatorname{Ext}_{R}^{1}(M, X) \longrightarrow 0 .
\end{aligned}
$$

Since $R$ is a graded isolated singularity, $\operatorname{dim}^{*} \operatorname{Ext}_{R}^{1}(M, X)$ is finite. Thus ${ }^{*} \operatorname{Ext}_{R}^{1}(M, X)_{ \pm l_{1}}=0$ for sufficiently large $l_{1} \gg 0$. Similarly there also exists an integer $l_{2} \gg 0$ so that ${ }^{*} \operatorname{Ext}_{R}^{1}(N, X)_{ \pm l_{2}}=0$.

Set $l=\max \left\{l_{1}, l_{2}\right\}$. Then we have

$$
\begin{aligned}
0 \longrightarrow{ }^{*} \operatorname{Hom}_{R}(M, X)_{ \pm l} & \longrightarrow{ }^{*} \operatorname{Hom}_{R}(M, E)_{ \pm l} \\
& \longrightarrow{ }^{*} \operatorname{Hom}_{R}\left(M, \Omega^{-1} X\right)_{ \pm l}
\end{aligned}
$$

Therefore, we obtain the equation

$$
[M, X( \pm l)]=[M, E( \pm l)]-\left[M, \Omega^{-1} X( \pm l)\right] .
$$


We also have

$$
[N, X( \pm l)]=[N, E( \pm l)]-\left[N, \Omega^{-1} X( \pm l)\right] .
$$

Suppose that $[M, X( \pm l)]<[N, X( \pm l)]$. Then the following inequality holds.

$$
[M, E( \pm l)]-\left[M, \Omega^{-1}(X)( \pm l)\right]<[N, E( \pm l)]-\left[N, \Omega^{-1} X( \pm l)\right] .
$$

Since $h(M)=h(N),[M, E( \pm l)]=[N, E( \pm l)]$. Hence, we see that $\left[M, \Omega^{-1} X( \pm l)\right]>\left[N, \Omega^{-1} X( \pm l)\right]$. This is a contradiction since $M \leq$ hom $N$. Therefore, we have some integer $l$ such that $[M, X( \pm l)]=$ $[N, X( \pm l)]$.

Remark 3.6. For a graded module $X, \Omega^{1} X$ is unique up to a free summand. Thus, $\Omega^{-1} X$ is not determined uniquely. Note that we can determine $\Omega^{1} X$; hence, $\Omega^{-1} X$, uniquely by taking it in a minimal graded free resolution since $R$ is ${ }^{*}$ local. However, we do not need a uniqueness of $\Omega^{-1} X$ to prove Lemma 3.5. We only use the fact that $\left[M, \omega_{R}(n)\right]=\left[N, \omega_{R}(n)\right]$ for each $n \in \mathbb{Z}$ if $h(M)=h(N)$.

We say that the category $\mathrm{CM}^{\mathbb{Z}}(R)$ is of graded finite representation type if there are only a finite number of isomorphism classes of indecomposable graded Cohen-Macaulay modules up to shift. We note that, if $\mathrm{CM}^{\mathbb{Z}}(R)$ is of finite representation type, then $R$ is a graded isolated singularity. See [15, Chapter 15] for details.

As an immediate consequence of Lemma 3.5, we have the following.

Corollary 3.7. Let $R$ be of finite representation type, and let $M$ and $N$ be graded Cohen-Macaulay R-modules. Assume that $h(M)=h(N)$ and $M \leq_{\text {hom }} N$. Then there are only finitely many indecomposable graded Cohen-Macaulay $R$-modules $X$ such that $[N, X]-[M, X]>0$.

For graded Cohen-Macaulay $R$-modules $M$ and $N$, we consider the following set of all the isomorphism classes of indecomposable graded Cohen-Macaulay modules

$$
\mathcal{F}_{M, N}=\{X \mid[N, X]-[M, X]>0\} / \cong .
$$

Note from Corollary 3.7 that $\mathcal{F}_{M, N}$ is a finite set if $h(M)=h(N)$ and $M \leq_{\text {hom }} N$. We also note that $\omega_{R} \notin \mathcal{F}_{M, N}$ if $h(M)=h(N)$. 
In the rest of this section we always assume that $R_{0}=k$ is an algebraically closed field.

Proposition 3.8. [14]. Let $R$ be a graded Gorenstein ring which is of graded finite representation type, and let $M$ and $N$ be graded CohenMacaulay R-modules. Assume that $h(M)=h(N)$ and $M \leq_{\text {hom }} N$. Then there exists some graded Cohen-Macaulay $R$-module $L$ such that $M \oplus L$ degenerates to $N \oplus L$.

Proof. Although a proof of the proposition is given in [14], we refer to the argument of the proof in the present paper. For this reason we briefly recall the proof.

Since $R$ is a graded isolated singularity, $\mathrm{CM}^{\mathbb{Z}}(R)$ admits AR sequences. For each $X \in \mathcal{F}_{M, N}$, we can take an AR sequence starting from $X$.

$$
\Sigma_{X}: 0 \longrightarrow X \longrightarrow E_{X} \longrightarrow \tau^{-1} X \longrightarrow 0 .
$$

Now we consider a sequence which is a direct sum of $[N, X]-[M, X]$ copies of $\Sigma_{X}$ where $X$ runs through all modules in $\mathcal{F}_{M, N}$. Namely,

$$
\bigoplus_{X \in \mathcal{F}_{M, N}} \Sigma_{X}^{[N, X]-[M, X]}=0 \longrightarrow U \longrightarrow V \longrightarrow W \longrightarrow 0
$$

For any indecomposable $Z \in \mathrm{CM}^{\mathbb{Z}}(R)$, we obtain

$$
\begin{aligned}
0 \longrightarrow \operatorname{Hom}_{R}(W, Z) \longrightarrow & \operatorname{Hom}_{R}(V, Z) \\
& \longrightarrow \operatorname{Hom}_{R}(U, Z) \longrightarrow k^{[N, Z]-[M, Z]} \longrightarrow 0 .
\end{aligned}
$$

This implies that the equality

$$
[U, Z]+[W, Z]-[V, Z]=[N, Z]-[M, Z],
$$

thus

$$
[U, Z]+[W, Z]+[M, Z]=[N, Z]+[V, Z]
$$


holds for all $Z \in \mathrm{CM}^{\mathbb{Z}}(R)$. Hence, this yields that $M \oplus U \oplus W \cong N \oplus V$. Since $V$ degenerates to $U \oplus W$, therefore $M \oplus V$ degenerates to $M \oplus U \oplus W \cong N \oplus V$.

Now we focus on the case that a graded Gorenstein ring is of graded finite representation type and representation directed. We say that a graded Cohen-Macaulay ring $R$ is representation directed if the AR quiver of $\mathrm{CM}_{0}^{\mathbb{Z}}(R)$ has no oriented cyclic paths. This notion was studied by Bongartz for finite-dimensional $k$-algebras in [6]. In our graded settings, similar results hold. Actually we shall prove following.

Theorem 3.9. Let $R$ be a graded Gorenstein ring which is of graded finite representation type and representation directed. Then the following conditions are equivalent for $M$ and $N \in \mathrm{CM}^{\mathbb{Z}}(R)$.

(1) $h(M)=h(N)$ and $M \leq_{\mathrm{hom}} N$.

(2) $M \leq_{\operatorname{deg}} N$.

(3) $M \leq \leq_{\operatorname{ext}} N$.

To prove the theorem, we modify the arguments in [6].

Lemma 3.10 (Cancellation property). Let $M, N$ and $X$ be finitely generated graded $R$-modules.

(1) Assume that $[X, M]=[X, N]$. If $M \oplus X$ gradually degenerates to $N \oplus X, M$ gradually degenerates to $N$.

(2) Assume that $R$ is Gorenstein and $M$ and $N$ graded CohenMacaulay $R$-modules. If $M$ gradually degenerates to $N \oplus F$ for some graded free $R$-module $F$, then $M / F$ gradually degenerates to $N$.

Proof. (1) Since $M \oplus X$ gradually degenerates to $N \oplus X$, there exist an exact sequence

$$
0 \longrightarrow W \longrightarrow M \oplus X \oplus W \longrightarrow N \oplus X \longrightarrow 0 .
$$


We construct a pushout diagram.

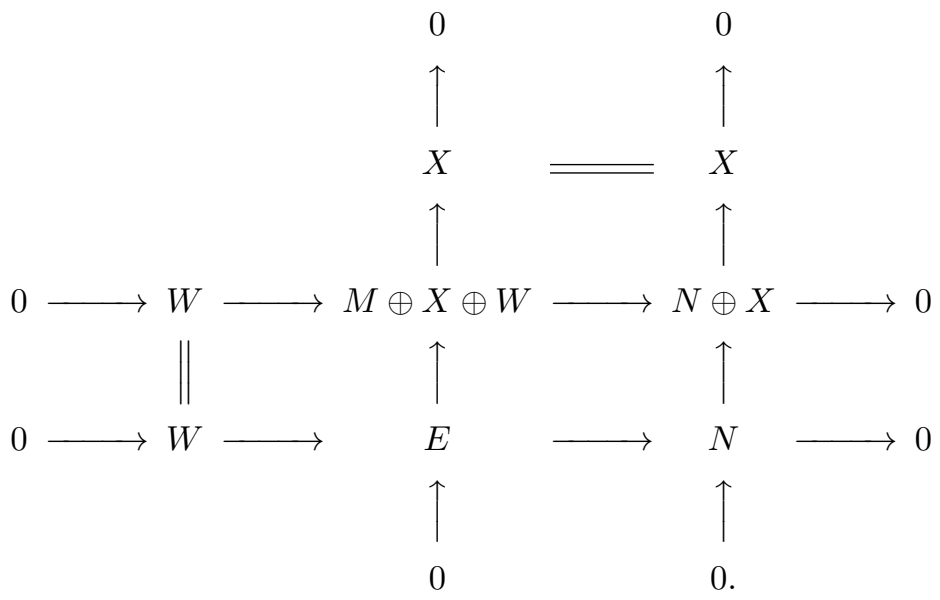

For the middle column sequence,

$$
[X, X]+[X, E]-[X, M \oplus X \oplus W]=[X, E]-[X, M]-[X, W] \geq 0
$$

On the other hand, for the bottom row sequence, since $[X, M]=[X, N]$,

$$
[X, N]+[X, W]-[X, E]=[X, M]+[X, W]-[X, E] \geq 0 .
$$

Thus, we have

$$
[X, X]+[X, E]-[X, M \oplus X \oplus W]=0
$$

This implies that the middle column sequence splits, so that $X \oplus E \cong$ $M \oplus X \oplus W$. Therefore, $E \cong M \oplus W$ and we get

$$
0 \longrightarrow W \longrightarrow M \oplus W \longrightarrow N \longrightarrow 0
$$

Namely, $M$ gradually degenerates to $N$.

(2) Since $M$ gradually degenerates to $N \oplus F$, we have an exact sequence

$$
0 \longrightarrow Z \longrightarrow M \oplus Z \longrightarrow N \oplus F \longrightarrow 0 \text {. }
$$

Suppose that $Z$ contains a graded free module $G$ as a direct summand. 
We construct a pushout diagram

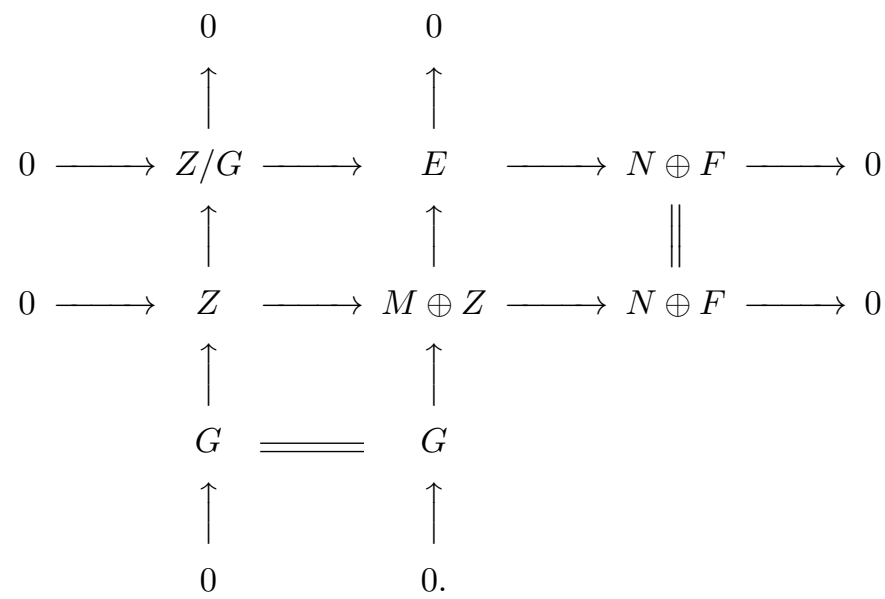

The left column sequence is a split sequence induced by the decomposition $Z \cong Z / G \oplus G$. Note that $E$ is also a graded Cohen-Macaulay module. Since $R$ is Gorenstein, the middle column sequence is also split. Hence,

$$
E \oplus G \cong M \oplus Z \cong M \oplus Z / G \oplus G .
$$

This yields that $E \cong M \oplus Z / G$. Hence, we may assume that $Z$ has no graded free modules as direct summands.

Consider a composition of the surjection $M \oplus Z \rightarrow N \oplus F$ and the projection $N \oplus F \rightarrow F$. Then the composition mapping is split, so that $M$ contains $F$ as a direct summand. Hence, $M \cong M / F \oplus F$ gradually degenerates to $N \oplus F$. Since $[F, M / F \oplus F]=[F, M]=[F, N \oplus F]$, by (1), we conclude that $M / F$ gradually degenerates to $N$.

For indecomposable graded Cohen-Macaulay modules $M$ and $N$, we write $X \preceq Y$ if $X \cong Y$ or if there exists a finite path from $X$ to $Y$ in the $\mathrm{AR}$ quiver of $\mathrm{CM}_{0}^{\mathbb{Z}}(R)$.

Lemma 3.11. Let $R$ be a graded Gorenstein ring which is of graded finite representation type and representation directed, and let $M$ and $N \in \mathrm{CM}^{\mathbb{Z}}(R)$. Assume that $h(M)=h(N), M \leq_{\text {hom }} N$ and $M$ and $N$ have no common direct summands. Let $X \in \mathrm{CM}^{\mathbb{Z}}(R)$ be an indecomposable module such that $\preceq$-minimal with the property $[N, X]-$ 
$[M, X]>0$ and $E$ be a middle term of an $A R$ sequence starting from $X$. Then $[E, N]=[E, M]$.

Proof. As in the proof of Proposition 3.8, we can construct the sequence

$$
0 \longrightarrow U \longrightarrow V \longrightarrow W \longrightarrow 0
$$

in $\mathrm{CM}^{\mathbb{Z}}(R)$ such that $U \oplus W \oplus M \cong V \oplus N$ via taking a direct sum of AR sequences starting from modules in $\mathcal{F}_{M, N}$. This isomorphism implies that $[Z, U]+[Z, W]-[Z, V]=[Z, N]-[Z, M]$ for each $Z \in \mathrm{CM}^{\mathbb{Z}}(R)$. Thus, it is enough to show that the equality $[E, U]+[E, W]-[E, V]=0$ holds. If there is a $Y \in \mathcal{F}_{M, N}$ such that, for the AR sequence $0 \rightarrow Y \rightarrow G \rightarrow \tau^{-1} Y \rightarrow 0$,

$$
[E, Y]+\left[E, \tau^{-1} Y\right]-[E, G]>0 .
$$

If $Y \cong X$, then one can show that $\tau^{-1} X$ is a direct summand of $E$, so that there is a cyclic path. This is a contradiction since $R$ is represented directed. Thus, $Y$ is not isomorphic to $X$. The inequality also shows that $\tau^{-1} Y$ is a direct summand of $E$. Thus, there is an irreducible map from $X$ to $\tau^{-1} Y$. Hence, $X$ is a direct summand of $G$, so that $Y \preceq X$. This is a contradiction. Consequently, for each $Y \in \mathcal{F}_{M, N}$, $[E, Y]+\left[E, \tau^{-1} Y\right]-[E, G]=0$, therefore $[E, N]=[E, M]$.

Proof of Theorem 3.9. The implication $(3) \Rightarrow(2) \Rightarrow(1)$ is trivial. Note that $\operatorname{Ext}_{R}^{1}(X, X)=0$ for an indecomposable $X \in \mathrm{CM}^{\mathbb{Z}}(R)$ since $R$ is representation directed. Thus, the implication $(2) \Rightarrow(3)$ can be shown by the same argument in $[\mathbf{2 0}, 3.5]$.

Now we shall show (1) $\Rightarrow(2)$. Set $V=\oplus_{W \in \mathcal{F}_{M, N} W \text {. Since }}$ $[N, X]-[M, X]=0$ for any $X \notin \mathcal{F}_{M, N}$, we can show the implication by induction on $d=[N, V]-[M, V]$. If $d=0,[N, Z]=[M, Z]$ for each $X \in \mathrm{CM}^{\mathbb{Z}}(R)$. Thus, $M \cong N$. Hence, assume that $d>0$ and $M$ and $N$ have no summand in common in the inductive step. We take $X \in \mathrm{CM}^{\mathbb{Z}}(R)$ in Lemma 3.11 and let $E$ be a middle term of the AR sequence starting from $X$. By virtue of Lemma 3.11 and Lemma 3.10 (1), it is enough to show that $E \oplus M \leq_{\operatorname{deg}} E \oplus N$. Now we have $E \oplus M \leq_{\text {ext }} X \oplus \tau^{-1} X \oplus M$. By the property of the AR sequence, for each indecomposable $Y$,

$$
[X, Y]+\left[\tau^{-1} X, Y\right]=[E, Y]+\delta_{X, Y},
$$


where $\delta_{X, Y}=1$ if $X \cong Y$ and otherwise 0 . Thus, $X \oplus \tau^{-1} X \oplus$ $M \leq_{\text {hom }} E \oplus N$. We should remark that $\mathcal{F}_{X \oplus \tau^{-1} X \oplus M, E \oplus N}$ is contained in $\mathcal{F}_{M, N}$ since $X \in \mathcal{F}_{M, N}$. Then

$$
\begin{aligned}
{[E \oplus N, V] } & -\left[X \oplus \tau^{-1} X \oplus M, V\right] \\
& =[N, V]-[M, V]+[E, V]-\left[X \oplus \tau^{-1} X, V\right] \\
& =d-1
\end{aligned}
$$

By the induction hypothesis,

$$
E \oplus M \leq_{\operatorname{ext}} X \oplus \tau^{-1} X \oplus M \leq_{\operatorname{deg}} E \oplus N
$$

so that $E \oplus M \leq_{\mathrm{deg}} E \oplus N$.

Remark 3.12. The implication $M \leq_{\operatorname{ext}} N \Rightarrow M \leq_{\operatorname{deg}} N$ does not hold in general. Let $R=k[x, y] /\left(x^{2}\right)$ with $\operatorname{deg} x=\operatorname{deg} y=1$. Then $R(-1)$ gradually degenerates to $\left(x, y^{2}\right) R$. In fact, we have an exact sequence

$$
\begin{aligned}
& 0 \longrightarrow R /(x)(-2) \stackrel{\left(\begin{array}{l}
x \\
0
\end{array}\right)}{\longrightarrow} R(-1) \oplus R /(x)(-2) \stackrel{\left(\begin{array}{ll}
x & y^{2}
\end{array}\right)}{\longrightarrow}\left(x, y^{2}\right) R \\
& \longrightarrow \quad 0 .
\end{aligned}
$$

Since $\left(x, y^{2}\right) R$ is an indecomposable graded Cohen-Macaulay module which is not isomorphic to $R(-1), R(-1) \leq_{\text {ext }}\left(x, y^{2}\right) R$ can never happen. See also [9, Remark 2.5].

Proposition 3.13. Let $R$ be a graded Gorenstein ring which is of graded finite representation type, and let $M$ and $N$ be graded CohenMacaulay $R$-modules. Assume that $h(M)=h(N)$ and $M \leq_{\text {hom }} N$. Then for each indecomposable non-free graded Cohen-Macaulay $R$-module $X$, we have the following equality.

$$
[N, X]-[M, X]=\left[\tau^{-1} X, N\right]-\left[\tau^{-1} X, M\right] .
$$

Proof. Under the assumption, as in the proof of Proposition 3.8, we can construct an exact sequence

$$
0 \longrightarrow U \longrightarrow V \longrightarrow W \longrightarrow 0
$$

in $\mathrm{CM}^{\mathbb{Z}}(R)$ such that $[U, X]+[W, X]-[V, X]=[N, X]-[M, X]$. It is enough to show that the equality $[U \oplus W, X]-[V, X]=\left[\tau^{-1} X, U \oplus\right.$ $W]-\left[\tau^{-1} X, V\right]$ holds for each indecomposable $X$. Moreover, the 
sequence is a direct sum of AR sequences, so we may assume that $0 \rightarrow U \rightarrow V \rightarrow W \rightarrow 0$ is an AR sequence starting from $U$. Let $X \in \mathrm{CM}^{\mathbb{Z}}(R)$ be indecomposable and non projective. By the property of an $\mathrm{AR}$ sequence, we have

$$
\begin{aligned}
{[U \oplus W, X]-[V, X] } & =\delta_{U, X} \quad\left[\tau^{-1} X, U \oplus W\right]-\left[\tau^{-1} X, V\right] \\
& =\delta_{W, \tau^{-1} X} .
\end{aligned}
$$

Since $W \cong \tau^{-1} U$, we can get the equality.

\section{Remarks on stable degenerations of graded Cohen-Macau-} lay modules. In this section, we consider the stable analogue of degenerations of graded Cohen-Macaulay modules.

Let $R$ be a graded Gorenstein ring where $R_{0}=k$ is a field, and let $V=k[[t]]$ with a trivial gradation and $K=k(t)$. Note that $R \otimes_{k} V$ and $R \otimes_{k} K$ are graded Gorenstein rings as well. Then $\underline{\mathrm{CM}}^{\mathbb{Z}}\left(R \otimes_{k} V\right)$ and $\mathrm{CM}^{\mathbb{Z}}\left(R \otimes_{k} K\right)$ are triangulated categories. We denote by $\mathrm{L}$ : $\underline{\mathrm{CM}}^{\mathbb{Z}}\left(R \otimes_{k} V\right) \rightarrow \underline{\mathrm{CM}}^{\mathbb{Z}}\left(R \otimes_{k} K\right)\left(\right.$ resp. $\left.\mathcal{R}: \underline{\mathrm{CM}}^{\mathbb{Z}}\left(R \otimes_{k} V\right) \rightarrow \underline{\mathrm{CM}}^{\mathbb{Z}}(R)\right)$ the triangle functor defined by the localization by $t$ (respectively, taking $\left.-\otimes_{V} V / t V\right)$. See also [18, Definition 4.1].

Definition 4.1. Let $\underline{M}, \underline{N} \in \underline{\mathrm{CM}}^{\mathbb{Z}}(R)$. We say that $\underline{M}$ stably degenerates to $\underline{N}$ if there exists a graded Cohen-Macaulay module $\underline{Q} \in \underline{\mathrm{CM}}^{\mathbb{Z}}\left(R \otimes_{k} \bar{V}\right)$ such that $\mathrm{\textrm {L }}(\underline{Q}) \cong M \otimes_{k} K$ in $\underline{\mathrm{CM}}^{\mathbb{Z}}\left(R \otimes_{k} K\right)$ and $\overline{\mathcal{R}}(\underline{Q}) \cong \underline{N}$ in $\underline{\mathrm{CM}}^{\mathbb{Z}}(R)$.

One can show the following characterization of stable degenerations similarly to the proof of [18, Theorem 5.1].

Theorem 4.2. The following conditions are equivalent for graded Cohen-Macaulay $R$-modules $M$ and $N$.

(1) $F \oplus M$ degenerates to $N$ for some graded free $R$-module $F$.

(2) There is a triangle in $\underline{\mathrm{CM}}^{\mathbb{Z}}(R)$

$$
\underline{Z} \longrightarrow \underline{M} \oplus \underline{Z} \longrightarrow \underline{N} \longrightarrow \underline{Z}[1] .
$$

(3) $\underline{M}$ stably degenerates to $\underline{N}$. 
Proof. We should note that the implication $(3) \Rightarrow(1)$. In our setting, $R \otimes_{k} V$ and $R \otimes_{k} K$ are ${ }^{*}$ local. Then graded projective $R \otimes_{k} V$ (respectively, $R \otimes_{k} K$ )-modules are graded free $R \otimes_{k} V$ (respectively, $\left.R \otimes_{k} K\right)$-modules. Hence, we can show the implication as in the Artinian case of the proof of [18, Theorem 5.1].

Remark 4.3. A theory of degenerations for derived categories has been studied in [11]. There it was shown that, for complexes $M$ and $N$ in the bounded derived category of a finite dimensional algebra, $M$ degenerates to $N$ if and only if there exists a triangle of the form which appears in the above theorem. Let $R$ be a graded Gorenstein ring with $R_{0}=k$ an algebraically closed field. As shown in $[\mathbf{1}, \mathbf{1 0}, \mathbf{1 2}]$, suppose that $R$ has a simple singularity. Then there exists a Dynkin quiver $Q$ such that we have a triangle equivalence

$$
\underline{\mathrm{CM}}^{\mathbb{Z}}(R) \cong \mathrm{D}^{b}(k Q),
$$

where $\mathrm{D}^{b}(k Q)$ is a bounded derived category of the category of finitely generated left modules over a path algebra $k Q$. By virtue of Theorem 4.2, we can describe the degenerations for $\mathrm{D}^{b}(k Q)$ in terms of the graded degenerations for $\mathrm{CM}^{\mathbb{Z}}(R)$. Since the graded ring $R$ is of graded finite representation type and representation directed, we have already seen them in Theorem 3.9.

Acknowledgments. The author express his deepest gratitude to Tokuji Araya and Yuji Yoshino for valuable discussions and helpful comments. The author also thanks the referee for his/her careful reading and helpful comments..

\section{REFERENCES}

1. T. Araya, Exceptional sequences over graded Cohen-Macaulay rings, Math. J. Okayama Univ. 41 (2001), 81-102.

2. M. Auslander and R.-O. Buchweitz, The homological theory of maximal Cohen-Macaulay approximations, Mem. Soc. Math. France 38 (1989), 5-37.

3. M. Auslander and I. Reiten, Almost split sequences for $\mathbb{Z}$-graded rings, in Singularities, representation of algebras, and vector bundles, Lect. Notes Math. 1273, Springer, Berlin, 1987.

4. __ Almost split sequences for abelian group graded rings, J. Algebra 114 (1988), 29-39. 
5. K. Bongartz, A generalization of a theorem of M. Auslander, Bull. Lond. Math. Soc. 21 (1989), 255-256.

6. $\_$On degenerations and extensions of finite-dimensional modules, Adv. Math. 121 (1996), 245-287.

7. W. Bruns and J. Herzog, Cohen-Macaulay rings, Cambr. Stud. Adv. Math. 39, Cambridge University Press, Cambridge, 1993.

8. M. Hashimoto, Auslander-Buchweitz approximations of equivariant modules, Lond. Math. Soc. Lect. 282, Cambridge University Press, Cambridge, 2000.

9. N. Hiramatsu and Y. Yoshino, Examples of degenerations of Cohen-Macaulay modules, Proc. Amer. Math. Soc. 141 (2013), 2275-2288.

10. O. Iyama and R. Takahashi, Tilting and cluster tilting for quotient singularities, Math. Ann. 356 (2013), 1065-1105.

11. B. Jensen, X. Su and A. Zimmermann, Degenerations for derived categories, J. Pure Appl. Alg. 198 (2005), 281-295.

12. H. Kajiura, K. Saito and A. Takahashi, Matrix factorization and representations of quivers, II, Type ADE case, Adv. Math. 211 (2007), 327-362.

13. G.J. Leuschke and R. Wiegand, Cohen-Macaulay representations, Math. Surv. Mono. 181, American Mathematical Society, Providence, RI, 2012.

14. C. Riedtmann, Degenerations for representations of quivers with relations, Ann. Sci. Ecole Norm. Sup. 19 (1986), 275-301.

15. Y. Yoshino, Cohen-Macaulay modules over Cohen-Macaulay rings, Lond. Math. Soc. Lect. 146, Cambridge University Press, Cambridge, 1990.

16. On degenerations of Cohen-Macaulay modules, J. Algebra 248 (2002), 272-290.

17. , On degenerations of modules, J. Algebra 278 (2004), 217-226.

18. Stable degenerations of Cohen-Macaulay modules, J. Algebra 332 (2011), 500-521.

19. G. Zwara, A degeneration-like order for modules, Arch. Math. 71 (1998), $437-444$.

20. , Degenerations for modules over representation-finite algebras, Proc. Amer. Math. Soc. 127 (1999), 1313-1322.

21. _ Degenerations of finite-dimensional modules are given by extensions, Compos. Math. 121 (2000), 205-218.

Department of general education, Kure National College of Technology, 2-2-11, Agaminami, Kure Hiroshima, 737-8506 Japan

Email address: hiramatsu@kure-nct.ac.jp 